\title{
Radar and Collision at Sea
}

\author{
from A. J. R. Tyrrell
}

Two practical measures might go a long way at the moment towards solving some of the questions raised by Captain F. J. Wylie in his paper (Vol. VI, p. 27 I) and discussed in the last number of the Journal (p. 202). The first is the provision of a suitable plotting device. It should not be too difficult to evolve a mechanism whereby a strip of transparent tracing material is passed across the face of the radar screen by means of two rollers (similar in operation to the echo-sounder paper passing over the plate in the modern sounding machine) in a direction parallel to the ship's course and at a speed representing that of the vessel and proportional to the scale of the radar picture in use. Plotting would be done by marking the ship's position and all other objects on the screen at short intervals and joining the consecutive positions of each object with a line. There would then be a continuous and up-to-date true plot of the area, which would be no more cluttered up with plotting than the actual area was with ships and objects.

Secondly, the actual use of the above plotting device on 'mock radar displays ' which could be operated by lecturers as required. This requires the production of extra apparatus at the various training establishments and a series of varying circumstances to reproduce on the mock screen, but such realistic practice is the finest way of training observers once they have grasped the basic fundamentals and capabilities of radar.

My own conception of a code of conduct while navigating in fog with radar is that while vessels are out of sound range (or perhaps a specified distance beyond this range) they should be considered to have equal rights with respect to standing on and giving way, whether or not they are in actual fact hampered.

A radar-equipped vessel having plotted another vessel and decided that risk of collision exists, should stand-on or give way by applying the crossing, end-on or overtaking rule as though both vessels were power driven. The alteration must be bold. If the 'radar giving way' vessel stands on for any reason (i.e. close proximity to land, hampered vessel, not equipped with radar, \&c.), the avoiding action will be taken in accordance with normal practice after making aural contact. The following general circumstances are then possible:

(a) Two vessels each with radar and both able to mancuvre with reasonable freedom will clear each other.

(b) A radar vessel meeting a vessel without radar or a hampered vessel will pass clear of her when she herself has not the 'radar right of way'. When she has the right of way, both ships will require to identify themselves and feel their way past each other in the normal manner.

Such rules for conduct are limited but could be applied on all occasions. They are simple and ought to give a basis for mutual understanding and some indication of when and how another vessel is likely to take action. The close-quarter situation would not be avoided altogether, but there should be fewer ships in close contact. By allowing certain radar vessels to get away clear, although it means limiting the movement of other radar vessels, the consequence of all ships trying to get out of each other's way, by the way they think best, is avoided. 